\title{
3 Research Square

\section{Carotid Ultrasonography as a Strategy to Optimize the Management of Cardiovascular Risk in Patients with Type 1 Diabetes: A Cohort Study}

\section{Laura Boswell}

Hospital Clinic de Barcelona https://orcid.org/0000-0002-8553-4030

\section{Tonet Serés-Noriega}

Hospital Clinic de Barcelona

\section{Alex Mesa}

Hospital Clinic de Barcelona

\section{Verónica Perea}

Hospital Universitari MútuaTerrassa: Hospital Universitari MutuaTerrassa

\section{Adriana Pané}

Hospital Clinic de Barcelona

\section{Clara Viñals}

Hospital Clinic de Barcelona

Jesús Blanco

Hospital Clinic de Barcelona

\section{Marga Giménez}

Hospital Clinic de Barcelona Irene Vinagre

Hospital Clinic de Barcelona

\section{Enric Esmatjes}

Hospital Clinic de Barcelona Ignacio Conget

Hospital Clinic de Barcelona

Antonio J. Amor (D AJAMOR@clinic.cat)

Hospital Clinic de Barcelona

\section{Research Article}

Keywords: Type 1 diabetes, carotid ultrasound, subclinical aterosclerosis, lipid profile, cardiovascular risk

Posted Date: October 28th, 2021

DOI: https://doi.org/10.21203/rs.3.rs-1015404/v1 
License: (c) (i) This work is licensed under a Creative Commons Attribution 4.0 International License. Read Full License 


\section{Abstract}

Background: Although cardiovascular disease (CVD) remains the leading cause of mortality in type 1 diabetes (T1D), the use of cardioprotective drugs is scarce. We aimed to evaluate the impact of carotid ultrasonography (US) on the improvement in cardiovascular risk factors (CVRFs) in T1D.

Methods. T1D patients without CVD meeting criteria for lipid treatment according to guidelines (age $\geq 40$ years, nephropathy and/or $\geq 10$ years of

diabetes duration with $\geq 1$ additional CVRFs) were included. The carotid-US group (US-G) underwent a standardized US protocol and CVRF assessment; recommendations were made according to subclinical atherosclerosis status. The control group (CG) followed usual clinical practice. Changes in CVRFs, specially statin use and LDL-cholesterol levels, at one year were analysed.

Results: A total of 318 patients were included (51.3\% female, mean age of 49.1 years and 25.5 years of diabetes duration): 211 in the US-G and 107 in the CG. Participants in the US-G had a higher baseline LDL-cholesterol than controls (114 vs. $102 \mathrm{mg} / \mathrm{dL} ; \mathrm{p}<0.001$ ). Lipid-lowering treatment was modified in $38.9 \%$ in the US-G and $6.5 \%$ in the CG $(p<0.001)$. At 1 year, the US-G was more frequently on statins, had lower LDL-cholesterol and $27 \%$ had stopped smoking ( $p<0.001$ for all). Changes were more pronounced in those with plaques $(p<0.001)$. In multivariate analyses adjusted for age, sex and other CVRFs, belonging to the US-G was independently associated with the intensification of lipid-lowering treatment (OR 10.47 [4.06-27.01]).

Conclusions: Carotid-US is independently associated with an intensification of lipid-lowering therapy in a high-risk T1D population.

\section{Background}

Cardiovascular disease (CVD) is still the leading cause of morbidity and mortality in subjects with type 1 diabetes (T1D) (1,2). Even with good metabolic control $\left(\mathrm{HbA}_{1 \mathrm{c}}<7 \%\right)$, death by CVD is 3 -fold higher than in the general population.(3) T1D-specific and non-specific CVD risk factors (CVRFs) such as albuminuria, obstetric complications, smoking, insulin-resistance or glycaemic variability, contribute to the atherosclerotic process and, eventually, to cardiovascular events in this population, underscoring the relevance of a comprehensive cardiovascular risk management(4-9). Compelling evidence supports the major role of LDL-cholesterol (LDL-c) levels on CVD in T1D. Namely, an increase of $40 \mathrm{mg} / \mathrm{dL}$ is associated with a $35-50 \%$ greater risk of CVD(10). Cardioprotective treatment, especially intensive lipidlowering therapy is recommended for most patients with T1D after 10 years of diabetes duration, presence of CVRFs or nephropathy(11-13).

One of the main limitations of CVD prevention guidelines in T1D is that many of the recommendations are extended from type 2 diabetes (T2D) even though physiopathology and underlying mechanisms of atherosclerosis in T1D remain poorly understood and differ from T2D(14). This together with a lack of 
reliable tools to identify those at higher cardiovascular risk contribute to a low degree of compliance of these recommendations, both from health care professionals and patients. Thus, in clinical practice, rates of patients receiving lipid-lowering therapy and reaching treatment goals are low(15-17). Alternative approaches such as imaging techniques could help overcome these limitations(18). Carotid ultrasonography (US) is a non-invasive way to study preclinical atherosclerosis, which is a strong predictor of cardiovascular events $(19,20)$. The presence of preclinical atherosclerosis is considered a very-high risk situation and high-intensity cardioprotective treatment is recommended(13). Previous studies have revealed increased carotid intima-media thickness (IMT) in T1D patients, compared to controls, even at young ages or with no additional CVRFs(21-23). Overall, preclinical CVD is more common and more extensive in the T1D population, even at early disease stages(24). Even so, whether the assessment of preclinical atherosclerosis could improve T1D management and CVD risk has not been previously studied.

Against this background, the aim of this study was to evaluate the impact of performing a carotid-US to study preclinical atherosclerosis on the improvement of CVRFs after 1 year, compared to routine clinical practice. As LDL-c is a major contributor to CVD in this population(10), a special focus was put on the changes in lipid profile and high-intensity lipid therapy.

\section{Methods}

\subsection{Study design and participants}

A cohort study in subjects with T1D followed in a specialized Diabetes Unit from a tertiary hospital in Spain was conducted. On June 2015, a specific protocol for CVD risk assessment which included a structured evaluation of CVRFs and a standardized carotid-US to assess subclinical atherosclerosis, was designed. From June 2015 to December 2018, a total of 318 patients were recruited: $n=221$ were selected for carotid-US and structured CVRF assessment (US group) and 107 patients who fulfilled the inclusion criteria and followed usual care according to existing clinical practice guidelines during the same period were selected as controls (control group).

Inclusion criteria were: T1D diagnosis, no previous personal history of CVD (coronary artery disease, ischemic stroke, peripheral vascular disease or heart failure) and high CVD risk according to the standards proposed by the main CVD prevention guidelines(11-13): (a) Age $\geq 40$ years, (b) T1D individuals with any stage of diabetic nephropathy regardless of age and (c) T1D individuals of any age, with at least 10 years of disease duration and one additional CVD risk factor. Additional risk factors were: diabetic retinopathy, active smoking habit, hypertension, dyslipidaemia (LDL-c $>160 \mathrm{mg} / \mathrm{dL}$ or on statin therapy), low high-density lipoprotein cholesterol (HDL-cholesterol) (defined as $<40 \mathrm{mg} / \mathrm{dL}$ in men and $<45 \mathrm{mg} / \mathrm{dL}$ in women), triglycerides $\geq 150 \mathrm{mg} / \mathrm{dL}$, history of premature CVD in first degree relatives(13), impaired hypoglycaemia awareness or a previous episode of severe hypoglycaemia in the last 2 years or former episode of preeclampsia/eclampsia. 
In the US group, modifications in cardioprotective treatment (lipid-lowering, antihypertensive and/or antiplatelet drugs) and lifestyle changes were recommended according to the preclinical atherosclerosis status. Lipid-lowering drugs (mainly statins) were initiated or uptitrated if carotid plaques were present (LDL-c goal $<100 \mathrm{mg} / \mathrm{dL}$ if one/two plaques were present; $\mathrm{LDL}-\mathrm{c}<70 \mathrm{mg} / \mathrm{dL}$ when $\geq 3$ plaques were observed or one plaque was at least $2.5 \mathrm{~mm}$ of height); and antiplatelets were initiated only if significant stenoses $(\geq 50 \%)$ were found. All the participants $(n=318)$ were evaluated at 1 year regarding changes in their CVD risk factors.

Criteria for T1D included past or present positivity for glutamic acid decarboxylase 65 , tyrosinephosphatase-like protein IA2 or insulin antibodies (only considered in insulin-naive patients), diabetic ketosis or ketoacidosis following the onset of classical hyperglycaemic symptoms and/or need for continuous insulin treatment. Patients with clinical suspicion of latent autoimmune diabetes in adults (LADA) or prior pancreatic diseases (pancreatitis, surgery) were excluded.

The study protocol was conducted according to the principles of the Declaration of Helsinki and approved by the Institution's Research Ethics Committee. All participants provided written informed consent.

\subsection{Clinical and laboratory measures}

Age, gender, history of diabetes complications and duration, smoking status and medical treatment (specially cardioprotective treatment such as lipid-lowering, antihypertensive and anti-platelet drugs) were recorded. Patients were weighted barefoot and in light clothing, using a calibrated electronic scale to the nearest $0.1 \mathrm{~kg}$. Height was measured using a wall-mounted calibrated stadiometer to the nearest $0.5 \mathrm{~cm}$. Body mass index (BMI) was calculated as weight in kilograms divided by square height in metres. Blood pressure was measured using a blood pressure monitor after a few minutes of rest.

Diabetic nephropathy was defined as persistently increased albumin-to-creatinine ratio $(\geq 30 \mathrm{mg} / \mathrm{g}$ confirmed on at least two out of three consecutive measurements) or estimated glomerular filtration rate (eGFR) $<60 \mathrm{ml} / \mathrm{min} / 1.73 \mathrm{~m}^{2}$ or the use of angiotensin converting enzyme inhibitors (ACEls) or angiotensin II receptor blockers (ARB) with no history of hypertension or CVD. Diabetic retinopathy was obtained from medical records and diagnosed by a specialized ophthalmologist. Hypertension was defined as repeated clinical systolic blood pressure (BP) $\geq 140 \mathrm{mmHg}$ and/or diastolic BP $\geq 90 \mathrm{mmHg}$ or active treatment with antihypertensive drugs. Severe hypoglycemia (defined as an episode of confirmed hypoglycemia that required third-party assistance for recovery), hypoglycemia unawareness (defined as a score $>3$ in the validated Spanish version of the Clarke Test(25)) and personal history of preeclampsia/eclampsia in any pregnancy were also registered.

Fasting blood and urine samples were analysed in the local laboratory with standardized assays to measure glucose, $\mathrm{HbA}_{1 \mathrm{c}}$, lipid profile (including total cholesterol, HDL-cholesterol and triglycerides; LDL-c was calculated with the Friedewald formula), creatinine and urinary albumin-to-creatinine ratio. The eGFR was obtained with the chronic kidney disease-epidemiology collaboration equation (CKD-EPI). $\mathrm{HbA}_{1 \mathrm{c}}$ 
values (Tosoh G8 Automated HPLC Analyser-Tosoh Bioscience Inc., South San Francisco, CA, USA-DCCT aligned, normal range 4-6\%) were obtained from medical records.

\subsection{Carotid B-mode ultrasound imaging (US group)}

Participants underwent sonographic assessment of both carotid arteries using linear array broadband 5 to $10 \mathrm{MHz}$ transducers (Acuson X300 and Acuson X700 [Siemens] US systems). Predefined and standardized imaging protocols to evaluate carotid intima-media thickness (IMT) and plaque presence were carried out as previously described.(26) Carotid plaques were visualized using B-mode and colour Doppler examinations in both longitudinal and transverse planes to account for circumferential asymmetry. Carotid plaques were defined as focal wall thickenings protruding into the arterial lumen by at least $50 \%$ compared to the surrounding IMT value, or when IMT thickness was $\geq 1.5 \mathrm{~mm}$, measured from the media adventitia interphase to the intima-lumen surface(27). All IMTs were measured off-line by the same trained endocrinologist (A.J.A.) using a semiautomatic software. Mean common carotid artery (CCA-IMT), mean-maximum common carotid artery, mean carotid bulb, mean-maximum carotid bulb, mean internal carotid artery and mean-maximum internal carotid artery IMTs were recorded, as well as the maximum height of carotid plaques. When carotid plaques were present, maximum-IMT equalled the highest plaque height. Peak systolic and end diastolic velocities were used to evaluate carotid stenosis and the planimetric area was measured at a transversal view when significant plaques (IMT >2.5 mm) were found. A mean CCA-IMT >75th percentile (adjusted for gender and age range) was defined according to a previous study in a Spanish population without CVD risk factors(28).

\subsection{Statistical analyses}

Data are presented as median and $25^{\text {th }}$ and $75^{\text {th }}$ percentiles, mean \pm SD or number (percentage). Normal distribution of continuous variables was evaluated with the Kolmogorov-Smirnov test.

Between-group differences in clinical, anthropometric and laboratory variables were assessed using unpaired Student's t-test, Mann-Whitney test and chi-square tests as appropriate. The differences between baseline and 1-year follow-up in CVRFs were assessed with paired tests (Student's t-test and Wilcoxon test for continuous variables; McNemar's test for categorical variables). To search for independent relationships between carotid-US (independent variable) and CVD risk profile (intensification [initiation or uptitration] of lipid-lowering therapy and LDL-c levels decrease $\geq 40 \mathrm{mg} / \mathrm{dL}$ at 1 year; dependent variables in model 1 and 2, respectively) logistic binary regression multivariable models were constructed. Both models included: age and gender, diabetes-related risk factors (duration of $\mathrm{T} 1 \mathrm{D}$, mean $\mathrm{HbA}_{1 \mathrm{c}}$ during the previous 5 years [as a marker of long-term glycaemic control, a factor closely related to CVD in T1D in previous studies], presence of retinopathy and nephropathy) as well non-specific CVD risk factors as hypertension, active smoking, BMI, LDL-C and eGFR. Finally, a sub-analysis in those with diabetic nephropathy was also performed.

Significance level was defined as a P-value <0.05. IBM SPSS Statistics 23.0 (SPSS, Inc; Chicago, Illinois) was used to perform the statistical analysis. 


\section{Results}

\subsection{Study population's characteristics}

A total of 318 patients were included (51.3\% female, mean age of $49.1 \pm 10.1$ years and 25.5 [20.5-32.4] years of diabetes duration). The overall characteristics of the study population are shown in Table 1. Patients selected for carotid-US were younger ( $48.1 \pm 9.5$ vs. $51.1 \pm 10.9$ years, $p=0.017$ ), more frequently on continuous subcutaneous insulin infusion (CSII) therapy ( $35.2 \%$ vs. $22.4 \%, p=0.021)$, and showed a worse lipid profile (in terms of LDL-c; $p<0.001$ ) than controls. There were no differences in classical CVRFs (smoking habit, prevalence of hypertension or BMI) nor in the presence of microvascular complications. Regarding US findings (only in US group), 76 (36\%) participants had at least one carotid plaque (20 [9.5\%] had $\geq 3$ plaques), 56 (26.5\%) had an enlarged IMT (CCA-IMT >p75 and no plaques), and 79 (37.7\%) had a normal carotid-US; with no differences between sexes (Supplemental figure 1).

\subsection{Changes on cardiovascular risk profile at 1 year across study groups}

Lipid-lowering treatment was modified (initiated or intensified) in $38.9 \%(n=82)$ of the participants in the US group vs. $6.5 \%(n=8)$ in the control group at 1 year $(p<0.001)$. Consequently, the US group were more frequently on statins after the study period (56.9\% at 1 year vs. $41.7 \%$ at baseline) and attained lower LDL-c values (102 vs. $114 \mathrm{mg} / \mathrm{dL} ; \mathrm{p}<0.001$ for all comparisons). Besides, the smoking habit was abandoned by $27 \%$ of former smokers in this group $(p<0.001)$. No clinically relevant changes were observed on glycaemic control, blood pressure or weight. Conversely, no differences in CVRFs were observed in the control group. Changes on cardiovascular risk factors are shown in table 2.

Logistic regression models were constructed to ascertain the independent association of carotid-US in the improvement of CVD risk profile. In addition to baseline age (OR 1.20 [1.00-1.44], per 5 years increments) and LDL-C (OR 1.21 [1.07-1.36], per $10 \mathrm{mg} / \mathrm{dL}$ increments), belonging to US group was the variable most strongly associated with the intensification of lipid-lowering treatment (OR 10.47 [4.06-27.01]; $p<0.001$; Table 3). Similarly, having undergone carotid-US was strongly associated with a decrease in LDL-c $\geq 40$ $\mathrm{mg} / \mathrm{dL}$ at 1 year (OR 4.69 [1.06-20.70]; $\mathrm{p}=0.043$; Supplemental Table 1).

\subsection{Changes on cardiovascular risk profile according to atherosclerosis status}

The CVD risk profile changes during the study period varied in relation to the findings in the carotid-US. In respect of the lipid profile, the changes were proportional to the atherosclerosis extent. Specifically, the intensity of lipid-lowering treatment and LDL-c levels were proportional to the extent of atherosclerosis revealed in the basal carotid-US ( $p$ for trend $<0.001$ ), as represented in Figure 1. Thus, the major changes occurred in the group with $\geq 3$ plaques: at baseline mean LDL-c was $115(92-122.5) \mathrm{mg} / \mathrm{dL}$ and $35 \%$ had an LDL-c $<100 \mathrm{mg} / \mathrm{dL}$. At one year, LDL-c was 84 (63.3-98.5) mg/dL and $80 \%$ were $<100 \mathrm{mg} / \mathrm{dL}(\mathrm{p}<0.001$ and $p=0.004$, respectively). Finally, in subjects without modification of the lipid-lowering treatment at 1 year, an improvement in LDL-c levels was observed in those with at least one carotid plaque $(n=11$; $L D L-c$ 
113 [90-137] vs. 97 [62-122] mg/dL, p=0.013; Supplemental Table 2). No clinically relevant changes were observed in patients without carotid plaques.

Interestingly, significant changes in smoking habits happened in the group with mean CCA-IMT $>$ p75 despite no plaques were present $(21.4 \%$ smokers at 1 year compared to $37.5 \%$ at baseline, $p=0.004)$. No clinically relevant changes in $\mathrm{HbA}_{1 \mathrm{c}}$, blood pressure or weight were observed, neither in the use of other cardioprotective drugs (ACEi/ARB or aspirin). Changes in CVRFs according to atherosclerosis status are detailed in Table 4.

\subsection{Sub-analysis of patients with diabetic nephropathy}

A total of 43 patients (55.8\% men, mean age $52.5 \pm 12$ years and 32 [27-38] years of diabetes duration) had diabetic nephropathy at baseline. Seventy percent also had concomitant diabetic retinopathy. Twenty-two were selected for carotid-US (31.8\% with $\geq 2$ carotid plaques and $36.4 \%$ with CCA-IMT $>$ p75) and 21 followed usual care. Patients selected for carotid-US were more often smokers, had higher LDL-c levels and were more often treated with ACEi or ARB, compared to the control group $(p<0.05$ for all comparisons; Table 5).

After 1 year, lipid-lowering treatment was optimized (started or intensified) in $45.5 \%(n=10)$ patients in the US group compared to $4.8 \%(n=1)$ in the control group $(p=0.002)$. Consequently, in the US group, LDL-c levels decreased 1 year after intervention ( $103.5 \mathrm{vs} .83 .5 \mathrm{mg} / \mathrm{dL}, \mathrm{p}=0.011)$ and more patients attained an LDL-c value $<100 \mathrm{mg} / \mathrm{dL}$ after the study period (36.4\% baseline vs. $69.8 \%$ at 1 year, $\mathrm{p}=0.039$ ). Again, results varied according to the US findings: in patients with carotid plaques, LDL-c significantly improved at 1 year (109 vs. $71 \mathrm{mg} / \mathrm{dL}, \mathrm{p}=0.018$ ), without changes in those without plaques. No significant changes were observed in the control group during follow-up.

\section{Discussion}

To our knowledge, this is the first study to describe an optimization of CVRFs driven by an intervention with a carotid-US exploration in a T1D population compared to usual care. Despite a worse baseline cardiovascular risk profile (higher LDL-c), subjects undergoing carotid-US, showed an improvement of their lipid profile (increased rates of statin treatment, lower LDL-c levels and increased rates of patients in treatment goals) and significant cessation of smoking habit, compared to routine care. Intensity of changes in the lipid profile were proportional to the atherosclerosis findings in the carotid-US, allowing a tailored approach of treatment goals. Altogether highlights carotid-US as a potential tool for focusing on CVD risk prevention, beyond glycaemic control.

In spite of significant advances in diabetes care and reduction of CVD in the past decades, patients with T1D still have an excess early CVD morbidity and mortality compared to the general population $(1,2)$. Cardiovascular risk increases in a stepwise fashion with each uncontrolled risk factor, even with good glycaemic control, underscoring the relevance of comprehensive cardiovascular risk management in this population(4). Although intensive lipid-lowering treatment is recommended for most 
patients with T1D (except young patients with short diabetes duration without additional CVRFs), in clinical practice, the rates of patients receiving these drugs and reaching treatment goals is insufficient(15-17). Our study suggests for the first time that carotid-US could help to improve overall management in T1D. Thus, performing carotid-US lead to a modification of lipid-lowering treatment in nearly $40 \%$ of patients, resulting in lower LDL-c which was maintained through the following year, while no differences were observed in patients following usual care. Since US is a simple and widely available procedure that does not involve radiation, it can be easily integrated in the endocrinology outpatient clinic. Considering that pragmatic clinical trials have confirmed the potential role of the visualization of the atherosclerotic process by US to improve overall CVD risk in primary prevention(29), this also could help to overcome the clinical inertia in CVD risk management in the T1D population.

Increasing age and LDL-c levels were independently related to the initiation or uptitration of lipid-lowering treatment at 1 year, in accordance with previous studies showing age, diabetes duration and LDL-c levels as CVRFs in $\operatorname{T1D}(2,17,30)$. Nevertheless, US-group allocation was the variable most strongly associated with intensification of lipid-lowering drugs, even after accounting for the former variables and others closely associated with CVD risk in the T1D population, such as traditional CVRFs or microvascular complications (Table 3). Further, undergoing carotid-US was also independently related with a more than 4.5 -fold chance of LDL-c $\geq 40 \mathrm{mg} / \mathrm{dL}(\approx 1 \mathrm{mmol} / \mathrm{L})$ decrease after 1 year, a secondary outcome that has been related to a significant decrease in CVD events in this population(10). Thus, US evaluation was associated not only with the initiation of lipid-lowering drugs, but also with improvement in lipoprotein profile to an extent that could be associated with hard clinical outcomes.

One of the main reasons for the low prevalence of statin treatment among T1D population could be the lack of a reliable instruments for selecting those most prone to suffer from future CVD(24). Although some clinical scores seem promising in this field $(31,32)$, more studies are needed to ascertain their usefulness in populations other than those where they were described(33). This poor information about CVD in T1D seems contradictory with the recent ESC/EASD guidelines, in which high-intensity statin treatment is recommended for most of the patients with T1D(11). In contrast with these rather uniform recommendations, carotid-US could be used to tailor CVD prevention in this population. In fact, our results showed a stepped increase in the intensification of the lipid-lowering treatment as the atherosclerosis burden increased. Moreover, one out of three of our pre-selected high-risk T1D patients did not show subclinical atherosclerosis, making unnecessary the initiation of intensified cardioprotective treatment (and, thus, avoiding possible side effects from these drugs). Altogether highlights the usefulness of US as a tool for the future precision medicine that we are heading towards.

Interestingly, patients with carotid plaques in whom no modification of the lipid-lowering therapy was made, improved their LDL-c levels at 1 year. This did not happen in patients without plaque or controls. This could be explained by and increased physicians' compromise and/or patient adherence after detecting the presence of subclinical atherosclerosis, improving overall treatment effectivity. This is in accordance with a study in general population that showed an improvement in CVD risk scores after 1 year of follow-up in patients who saw a pictorial representation of their carotid-US results and these were 
explained by a healthcare professional(29). Hence, carotid-US could also help to overcome nonadherence to cardioprotective drugs, a very important problem specially in primary prevention(34).

Another remarkable finding is that nearly one third of patients in the US group quitted smoking. This change was notable in those with no plaque but enlarged IMT. This group benefited from the intervention despite no changes in lipid profile at 1 year, but a reduced smoking rate, extending the benefits of subclinical atherosclerosis assessment. Smoking habit in T1D significantly and independently contributes to nephropathy progression(35), coronary heart disease and stroke(6). Strategies to reduce smoking habit are needed in clinical practice, especially in high-risk populations.

Nephropathy is a threatening complication of T1D. It frequently coexists with poor glycaemic control and classical CVRFs such as dyslipidaemia and smoking, which, in turn contribute to its development and progression(36,37). Nephropathy confers an extreme risk of CVD(11). Previous studies have shown that patients with T1D and nephropathy have higher plaque burden compared to those with normoalbuminuria(5).

In our sample, $13.5 \%$ had diabetic nephropathy. A subgroup analysis was performed in this extreme risk population. More than two thirds had an enlarged IMT or carotid plaques and all patients with plaques had a high plaque burden, in line with previous studies(5). Treatment was intensified in nearly half of the patients in the carotid-US group. Even with a worse initial cardiovascular profile (more smokers, higher LDL-C and higher rates of treatment with ACEi/ARB), patients selected for carotid-US had significantly lower LDL-c levels after 1 year and a higher proportion of patients under treatment goals (with no changes in the control group). Thus, performing carotid-US not only improves risk profile in general T1D population, but also in those specially at very-high risk.

Several strengths and limitations should be acknowledged. One of the main strengths is that, to our knowledge, this is the first study to describe an optimization of CVD risk profile after performing a carotidUS for the study of subclinical atherosclerosis in T1D. Likewise, this study included a control group following routine clinical practice, strengthening the relevance of our findings. Furthermore, in addition to IMT, carotid plaque was evaluated, which is a better predictor of CVD events, illustrating the robustness of our findings(38). Finally, a standardized CVD risk assessment protocol was performed in our study, limiting potential bias. Yet, our study also has some limitations. Of most relevance, its observational design precludes drawing conclusions on causality. Second, inclusion criteria were strict and only high CVD risk patients were selected, which may not be representative of the general T1D population. Third, nearly one third of patients fulfilling criteria for diabetic nephropathy were not receiving ACEi/ARB treatment, this was observed in up to $40 \%$ in patients not selected for carotid-US. Thus, initial stages of diabetic nephropathy still frequently go unrecognized/undertreated by endocrinologists and/or patients with diabetes. Additionally, this underscores the importance of comprehensive cardiovascular risk assessment (including nephropathy diagnosis and management) in this high-risk population in order to tackle CVD. Finally, the intensification of cardioprotective treatment according to US findings was not fully standardized. However, some pragmatic randomized trials using US as a visual tool for improving 
CVD risk in general population did not protocolize the treatment either, showing similar results(29). This underscores the usefulness of carotid-US evaluation in real clinical practice and highlights the need to protocolise patient management and treatment after weighing in carotid-US findings.

\section{Conclusions}

In summary, in high-risk T1D patients, assessing preclinical atherosclerosis using carotid-US was independently associated with an improvement in LDL-c levels, higher rates of patients in treatment goals and significant cessation of smoking habit after 1 year, compared to routine clinical care. This is the first study to assess the usefulness of this strategy. While our results need be replicated in other populations and awaiting longitudinal studies with hard CVD outcomes, carotid-US is a simple, accessible and visual tool that can help tailor cardiovascular risk and personalize CVD prevention strategies in T1D.

\section{Abbreviations}

ACEls: angiotensin converting enzyme inhibitors

ARB: angiotenisin II receptor blockers

BMI: body mass index

BP: blood pressure

CCA-IMT: Mean common carotid artery

CG: control group

CKD-EPI: chronic kidney disease-epidemiology collaboration equation

CSII: continuous subcutaneous insulin infusion

CVD: Cardiovascular disease

CVRFs: cardiovascular risk factors

IMT: intima-media thickness

LADA: latent autoimmune diabetes in adults

T1D: type 1 diabetes

T2D: type 2 diabetes

US: ultrasound 
US-G: carotid-US group

\section{Declarations}

\section{ETHICS APPROVAL AND CONSENT TO PARTICIPATE}

The study was approved by the Institution's Research Ethics Committee. All participants provided written informed consent.

\section{CONSENT FOR PUBLICATION}

Not applicable.

\section{AVAILABILITY OF DATA AND MATERIALS}

The datasets used during the current study are available from the corresponding author on reasonable request.

\section{COMPETING INTERESTS}

The authors declare that they have no competing interests.

\section{FUNDING}

No specific funding was received.

\section{AUTHORS' CONTRIBUTION}

L.B, T.S., A.M., V.P., A.P., C.V., MG., I.V., J.B., E.E., I.C. and A.J.A acquired and processed all clinical data. A.J.A performed the US measurements. L.B., A.J.A. and I.C. contributed to the study concept and design. All authors participated in data analysis and interpretation and reviewed the final version of the manuscript. L.B and A.J.A wrote the manuscript, designed the figures and had final responsibility for the decision to submit for publication. L.B. and A.J.A. are the guarantors of this work and, as such, had full access to all the data in the study and take responsibility for the integrity of the data and the accuracy of the data analysis.

\section{ACKNOWLEDGEMENTS}

A.J.A. received a research grant ("Ajut per a la recerca en diabetis modalitat clínica 2018") from the Associació Catalana de Diabetis (ACD). L.B received a research grant (Resident Award "Premi Fi de Residènica Emili Letang" 2019) from Hospital Clínic de Barcelona, Research, Innovation and Education Department and a research grant ("Ajut ACD per la realització del programa de doctorat 2020") from ACD.

\section{References}


Table 1. Clinical and biochemical characteristics of study subjects 


\begin{tabular}{|c|c|c|c|c|}
\hline & $\begin{array}{l}\text { Total } \\
\text { population } \\
(\mathrm{n}=318)\end{array}$ & $\begin{array}{l}\text { Carotid-US } \\
\text { group }(n=211)\end{array}$ & $\begin{array}{l}\text { Control } \\
\text { group } \\
(n=107)\end{array}$ & $\mathrm{P}$ \\
\hline \multicolumn{5}{|l|}{ Clinical characteristics } \\
\hline Age (years) & $49.1 \pm 10.1$ & $48.1 \pm 9.5$ & $51.1 \pm 10.9$ & 0.017 \\
\hline Gender (female) & $163(51.3)$ & $104(49.3)$ & $59(55.1)$ & 0.324 \\
\hline Current smokers & $80(25.2)$ & $56(26.5)$ & $24(22.4)$ & 0.425 \\
\hline Hypertension & $81(25.5)$ & $57(27)$ & $24(22.4)$ & 0.375 \\
\hline SBP $(m m H g)$ & $128(120-137)$ & $127(118-137)$ & $\begin{array}{l}130(120- \\
137.3)\end{array}$ & 0.207 \\
\hline $\mathrm{DBP}(\mathrm{mmHg})$ & $80(74-85)$ & $80(74-86)$ & $80(75.8-80)$ & 0.154 \\
\hline $\mathrm{BMI}\left(\mathrm{kg} / \mathrm{m}^{2}\right)$ & $\begin{array}{l}25.4(23.2- \\
28.4)\end{array}$ & $\begin{array}{l}25.7(23.6- \\
28.7)\end{array}$ & $\begin{array}{l}24.7(22.8- \\
27.6)\end{array}$ & 0.058 \\
\hline Duration of Diabetes (years) & $\begin{array}{l}25.5(20.5- \\
32.4)\end{array}$ & $25.7(22-31.2)$ & $\begin{array}{l}24.3(14.9- \\
37.4)\end{array}$ & 0.240 \\
\hline $\begin{array}{l}\text { Severe hypoglycaemia or impaired } \\
\text { hypoglycaemia awareness }\end{array}$ & $83(26.1)$ & $55(26.2)$ & $28(26.2)$ & 0.997 \\
\hline Diabetic retinopathy & $122(38.4)$ & $80(37.9)$ & $42(39.3)$ & 0.817 \\
\hline Proliferative retinopathy & $42(13.2)$ & $25(11.8)$ & $17(15.9)$ & 0.308 \\
\hline Diabetic nephropathy & $33(10.4)$ & $21(10)$ & $12(11.2)$ & 0.727 \\
\hline CSII & $98(30.8)$ & $74(35.1)$ & $24(22.4)$ & 0.021 \\
\hline \multicolumn{5}{|l|}{ Biochemical characteristics } \\
\hline $\mathrm{HbA}_{1 \mathrm{c}}(\%)$ & $7.5(7-8.1)$ & $7.5(7-8.1)$ & $7.6(7-8.1)$ & 0.951 \\
\hline Glucose (mg/dL) & $148(108-203)$ & $\begin{array}{l}148(106- \\
204.5)\end{array}$ & $\begin{array}{l}149(110.8- \\
201.3)\end{array}$ & 0.608 \\
\hline Alanine aminotransferase (UI/L) & $19(15-26))$ & $19.5(16-26)$ & $18(13-24)$ & 0.041 \\
\hline Leucocyte count (per $\mathrm{mm}^{3}$ ) & $\begin{array}{l}6.65(5.64- \\
8.16)\end{array}$ & $\begin{array}{l}6.68(5.67- \\
8.18)\end{array}$ & $\begin{array}{l}6.62(5.6- \\
8.1)\end{array}$ & 0.420 \\
\hline eGFR & $\begin{array}{l}96.7(85- \\
105.6)\end{array}$ & $99.9(88.4-107)$ & $\begin{array}{l}93.7(80.8- \\
103.4)\end{array}$ & 0.002 \\
\hline Total cholesterol (mg/dL) & $\begin{array}{l}185(169- \\
208.8)\end{array}$ & $\begin{array}{l}190(174- \\
213.3)\end{array}$ & $\begin{array}{l}177(158.8- \\
200)\end{array}$ & $<0.001$ \\
\hline Triglycerides (mg/dL) & $73(57-96.5)$ & $73(57-99.3)$ & $\begin{array}{l}72.5(57- \\
94.3)\end{array}$ & 0.958 \\
\hline LDL-c (mg/dL) & $109(95-126)$ & $114(100-131)$ & $102(88-115)$ & $<0.001$ \\
\hline
\end{tabular}




\begin{tabular}{|lllll|}
\hline \multicolumn{1}{|c}{ LDL-c $<100 \mathrm{mg} / \mathrm{dL}$} & $106(33.3)$ & $55(26.1)$ & $51(47.7)$ & $<0.001$ \\
$\mathrm{LDL}-\mathrm{c}<70 \mathrm{mg} / \mathrm{dL}$ & $9(2.8)$ & $1(0.5)$ & $8(7.5)$ & $<0.001$ \\
\hline HDL-c $(\mathrm{mg} / \mathrm{dL})$ & $61(49-72)$ & $59(50-70)$ & $60(48-75)$ & 0.830 \\
\hline Non-HDL-c $(\mathrm{mg} / \mathrm{dL})$ & $126(109-141)$ & $\begin{array}{l}130.5(113.8- \\
149.3)\end{array}$ & $\begin{array}{l}115.5(102.8- \\
128.3)\end{array}$ & $<0.001$ \\
\hline TC/HDL-c ratio & $3.1(2.7-3.8)$ & $3.3(2.7-3.8)$ & $2.9(2.5-3.4)$ & 0.001 \\
\hline Pharmacological treatment & & & & \\
\hline Statin Use & $125(39.3)$ & $88(41.7)$ & $37(34.6)$ & 0.219 \\
\hline ACEi/ARB & $86(27)$ & $62(29.4)$ & $24(22.4)$ & 0.187 \\
\hline Aspirin & $31(9.7)$ & $17(8.1)$ & $14(13.1)$ & 0.153 \\
\hline
\end{tabular}

Values expressed as mean \pm SD, median (interquartile range) or number (percentage). P-value between Carotid-US vs. control groups are represented.

ACEi: angiotensin-converting-enzyme inhibitor; ARB: angiotensin II receptor blocker; BMI: body mass index; CSII: Continuous subcutaneous insulin infusion; CVD: cardiovascular disease; DBP: diastolic blood pressure; eGFR: estimated glomerular filtration rate; HDL-c: high-density lipoprotein cholesterol; LDL-C: low-density lipoprotein cholesterol; SBP: systolic blood pressure; TC: total cholesterol; US: ultrasonography

Table 2. Changes on cardiovascular risk factors 1 year after the intervention across study groups 
Baseline

\section{Active smokers}

Control group

Carotid-US group

\section{Statin use}

Control group

Carotid-US group

\section{Ezetimibe use}

Control group

Carotid-US group

\section{LDL-c (mg/dL)}

Control group

Carotid-US group

LDL-c $<100 \mathrm{mg} / \mathrm{dL}$

Control group

Carotid-US group

LDL-c $<70 \mathrm{mg} / \mathrm{dL}$

Control group

Carotid-US group

$\mathrm{HbA}_{1 \mathrm{c}}(\%)$

Control group

Carotid-US group

\section{SBP (mmHg)}

Control group

Carotid-US group

Weight (kg)

Control group

Carotid-US group

AAS use

$73 \pm 13.2$

$75.2 \pm 14.8$
1 year

P

24 (22.4)

22 (20.6)

1.000

$56(26.5)$

41 (19.4)

$<0.001$

37 (34.6)

41 (38.3)

0.219

88 (41.7)

120 (56.9)

$<0.001$

$5(4.7)$

$6(5.6)$

1.000

5 (2.4)

$11(5.2)$

0.109

98 (85-107)

0.185

102 (83-122)

$<0.001$

114 (100-131)

63 (58.9)

0.104

99 (46.9)

$<0.001$

55 (26.1)

7 (6.7)

1.000

$19(9.2)$

$<0.001$
$7.5(6.9-8.1)$

0.609

$7.5(7.0-8.1)$

0.049
130.5 (121-140)

0.064

127 (118-137)

130 (122-135.5)

0.033

$\begin{array}{lll}130(120-137.5) & 130.5(121-140) & 0.064 \\ 127(118-137) & 130(122-135.5) & 0.033\end{array}$

$72.8 \pm 13.9$

0.531

$75.3 \pm 14.9$

0.737 


\begin{tabular}{|cccc|}
\hline Control group & $14(13.1)$ & $14(13.1)$ & 1.000 \\
\hline Carotid-US group & $17(8.1)$ & $21(10)$ & 0.289 \\
\hline ACEi/ARB use & $24(22.4)$ & $25(23.4)$ & 1.000 \\
\hline Control group & $62(19.4)$ & $58(27.5)$ & 0.454 \\
\hline Carotid-US group & & \\
\hline $\begin{array}{l}\text { Control group: n=107, Carotid-US group: n=211. } \\
\text { AAS: acetylsalicylic acid; ACEi: angiotensin-converting-enzyme inhibitor; ARB: angiotensin II receptor } \\
\text { blocker; LDL-C: low-density lipoprotein cholesterol; SBP: systolic blood pressure }\end{array}$ & \\
\hline
\end{tabular}

Table 3. Logistic regression model assessing the baseline factors associated with initiation/increasing dose of lipid-lowering treatment at 1 year.

\begin{tabular}{|lll|}
\hline & OR (95\% Cl) & p-value \\
\hline Age (per 5 years increment) & $1.20(1.00-1.44)$ & 0.050 \\
\hline Gender (male) & $1.14(0.63-2.05)$ & 0.655 \\
\hline Hypertension & $1.04(0.50-2.14)$ & 0.922 \\
\hline Active smoking habit & $1.47(0.76-2.84)$ & 0.252 \\
\hline Body mass index (kg/m2) & $1.00(0.93-10.7)$ & 0.990 \\
\hline Diabetic nephropathy & $1.48(0.51-4.24)$ & 0.469 \\
\hline Diabetic retinopathy & $0.77(0.38-1.52)$ & 0.449 \\
\hline Diabetes duration (years) & $1.01(0.98-1.05)$ & 0.560 \\
\hline LDL-c (per 10 mg/dL increment) & $1.21(1.07-1.36)$ & 0.003 \\
\hline eGFR (ml/min) & $0.98(0.96-1.00)$ & 0.112 \\
\hline HbA1c (\%) & $0.96(0.69-1.33)$ & 0.816 \\
\hline US-group allocation & $10.47(4.06-27.01)$ & $<0.001$ \\
\hline
\end{tabular}

Table 4. Changes in cardiovascular risk factors according to atherosclerosis status in the US group $(n=211)$ 


\begin{tabular}{|c|c|c|c|}
\hline & Baseline & 1 year & $\mathbf{P}$ \\
\hline \multicolumn{4}{|l|}{ Smoking habit } \\
\hline Normal & $16(20.3)$ & $11(13.9)$ & 0.063 \\
\hline Mean CCA-IMT > p75 & $21(37.5)$ & $12(21.4)$ & 0.004 \\
\hline 1-2 plaques & $11(19.6)$ & $10(17.9)$ & 1.00 \\
\hline$\geq 3$ plaques & $8(40)$ & $8(40)$ & 1.00 \\
\hline \multicolumn{4}{|l|}{ LDL-c (mg/dL) } \\
\hline Normal & 112 (99-132) & $110(93.8-126.8)$ & 0.235 \\
\hline Mean CCA-IMT > p75 & $107.5(99.3-130.5)$ & $104(88.5-124-3)$ & 0.040 \\
\hline 1-2 plaques & $120(103.5-134.8)$ & $89(72-117)$ & $<0.001$ \\
\hline$\geq 3$ plaques & $115(92-122.5)$ & $84(63.3-98.5)$ & $<0.001$ \\
\hline \multicolumn{4}{|l|}{$\mathrm{HbA}_{1 \mathrm{c}}(\%)$} \\
\hline Normal & $7.4(7-8)$ & $7.5(6.9-8)$ & 0.245 \\
\hline Mean CCA-IMT > p75 & $7.7(7.3-8.1)$ & $7.4(7-8.2)$ & 0.257 \\
\hline 1-2 plaques & $7.5(7-8.1)$ & $7.5(7-8.1)$ & 0.387 \\
\hline$\geq 3$ plaques & $7.4(6.8-8.2)$ & $7.4(6.8-7.8)$ & 0.615 \\
\hline \multicolumn{4}{|l|}{ SBP $(\mathrm{mmHg})$} \\
\hline Normal & $123(113-131)$ & $128(120-135)$ & 0.017 \\
\hline Mean CCA-IMT > p75 & $127(120-137.8)$ & $130(123-133.5)$ & 0.624 \\
\hline 1-2 plaques & $132(119.3-139.5)$ & $130(125-140)$ & 0.336 \\
\hline$\geq 3$ plaques & $135(120-145)$ & $135(118.5-140)$ & 0.641 \\
\hline \multicolumn{4}{|l|}{ Weight (kg) } \\
\hline Normal & $74.4 \pm 14.3$ & $74.7 \pm 14.7$ & 0.441 \\
\hline Mean CCA-IMT > p75 & $78.3 \pm 15.3$ & $78.3 \pm 14.7$ & 0.877 \\
\hline 1-2 plaques & $73.1 \pm 14.1$ & $73 \pm 13.9$ & 0.840 \\
\hline$\geq 3$ plaques & $76.1 \pm 16.5$ & $76.1 \pm 18.2$ & 0.979 \\
\hline
\end{tabular}

Data are shown as mean \pm standard deviation, median (interquartile range) or $\mathrm{n}$ (percentage). P-values between baseline vs. 1-year follow-up are presented.

Normal US: $n=79$; Mean CCA-IMT >p75: $n=56 ; 1-2$ plaques: $n=56$; $\geq 3$ plaques: $n=20$ 
CCA: common carotid artery; IMT: intima-media thickness.

Table 5. Clinical and biochemical characteristics of the patients with diabetic nephropathy 


\begin{tabular}{|c|c|c|}
\hline $\begin{array}{l}\text { All patients with } \\
\text { nephropathy }(n=43)\end{array}$ & $\begin{array}{l}\text { Carotid-US } \\
\text { group }(n=22)\end{array}$ & $\begin{array}{l}\text { Control } \\
\text { group } \\
(n=21)\end{array}$ \\
\hline
\end{tabular}

\section{Clinical characteristics}

\begin{tabular}{|c|c|c|c|c|}
\hline Age (years) & $52.5 \pm 12$ & $49.2 \pm 10.5$ & $56 \pm 12.5$ & 0.060 \\
\hline Gender (female) & $19(44.2)$ & $12(54.5)$ & 7 (33.3) & 0.161 \\
\hline Current smokers & $15(34.9)$ & $12(54.5)$ & $3(14.3)$ & 0.021 \\
\hline Hypertension & $24(55.8)$ & $14(63.6)$ & $10(47.6)$ & 0.290 \\
\hline SBP (mmHg) & $130(120-137)$ & $\begin{array}{l}126.5(118- \\
136.3)\end{array}$ & $\begin{array}{l}130(124- \\
140)\end{array}$ & 0.180 \\
\hline $\mathrm{DBP}(\mathrm{mmHg})$ & $80(75-81)$ & $\begin{array}{l}77(72.5- \\
87.8)\end{array}$ & $\begin{array}{l}80 \text { (76.5- } \\
80)\end{array}$ & 0.951 \\
\hline $\mathrm{BMI}\left(\mathrm{kg} / \mathrm{m}^{2}\right)$ & $26.7(24.3-29.2)$ & $\begin{array}{l}27.4(24.9- \\
28.8)\end{array}$ & $\begin{array}{l}26.5(23.8- \\
30.8)\end{array}$ & 0.688 \\
\hline Duration of Diabetes (years) & $32(27-38)$ & $\begin{array}{l}30.4(25.4- \\
36.8)\end{array}$ & $\begin{array}{l}34.4(27.1- \\
48.8)\end{array}$ & 0.132 \\
\hline $\begin{array}{l}\text { Severe hypoglycaemia or impaired } \\
\text { hypoglycaemia awareness }\end{array}$ & $9(21)$ & $3(13.6)$ & $6(28.6)$ & 0.229 \\
\hline Diabetic retinopathy & $30(69.8)$ & $13(59.1)$ & $17(81)$ & 0.119 \\
\hline Proliferative retinopathy & $17(39.5)$ & $8(36.4)$ & $9(42.9)$ & 0.638 \\
\hline CSII & $14(32.6)$ & $9(40.9)$ & $5(23.8)$ & 0.232 \\
\hline \multicolumn{5}{|l|}{ Biochemical characteristics } \\
\hline $\mathrm{HbA}_{1 \mathrm{c}}(\%)$ & $7.8(7.2-8.4)$ & $8(7.4-8.4)$ & $7.4(7-8.2)$ & 0.165 \\
\hline eGFR & $73.2(56-96)$ & $78(65-103)$ & $69(46-91)$ & 0.198 \\
\hline Total cholesterol (mg/dL) & $178(152-192)$ & $\begin{array}{l}182(159- \\
203)\end{array}$ & $\begin{array}{l}162(138.5- \\
189.5)\end{array}$ & 0.048 \\
\hline Triglycerides (mg/dL) & $76(55-100)$ & $\begin{array}{l}81(63.5- \\
108.5)\end{array}$ & $\begin{array}{l}75(54.5- \\
91)\end{array}$ & 0.375 \\
\hline LDL-c (mg/dL) & $101(80-114)$ & $\begin{array}{l}103.5(94.5- \\
125.5)\end{array}$ & $\begin{array}{l}86(70- \\
107.5)\end{array}$ & 0.009 \\
\hline LDL-c $<100 \mathrm{mg} / \mathrm{dL}$ & $21(48.8)$ & \multirow{2}{*}{$\begin{array}{l}8(36.4) \\
1(4.5)\end{array}$} & \multirow{2}{*}{$\begin{array}{l}13(61.9) \\
5(23.8)\end{array}$} & 0.094 \\
\hline LDL-c $<70 \mathrm{mg} / \mathrm{dL}$ & $6(14)$ & & & 0.068 \\
\hline HDL-c (mg/dL) & $55(42-68)$ & $58(44-68.5)$ & $53(38-67)$ & 0.224 \\
\hline
\end{tabular}




\begin{tabular}{|llccc|}
\hline Statin Use & $27(62.8)$ & $14(63.6)$ & $13(61.9)$ & 0.907 \\
\hline ACEi/ARB & $33(76.7)$ & $21(95.5)$ & $12(57.1)$ & 0.003 \\
\hline Aspirin & $13(30.2)$ & $5(22.7)$ & $8(38.1)$ & 0.273 \\
\hline US findings (only Carotid-US group) & & & & \\
\hline Normal & - & $7(31.8)$ & - & - \\
CCA-IMT >p75 (without plaques) & - & $8(36.4)$ & - & - \\
At least 1 carotid plaque & - & $7(31.8)$ & - & \\
$\geq 2$ plaques & & $3(23.6)$ & & \\
$\geq 3$ plaques & & $4(18.2)$ & \\
\hline
\end{tabular}

Values expressed as mean $\pm S D$, median (interquartile range) or number (percentage). P-value between Carotid-US vs. control groups are represented.

ACEi: angiotensin-converting-enzyme inhibitor; ARB: angiotensin II receptor blocker; BMI: body mass index; CCA: common carotid artery; CSII: Continuous subcutaneous insulin infusion; DBP: diastolic blood pressure; eGFR: estimated glomerular filtration rate; HDL-c: high-density lipoprotein cholesterol; IMT: intima-media thickness; LDL-c: low-density lipoprotein cholesterol; SBP: systolic blood pressure; TC: total cholesterol; US: ultrasonography

\section{Figures}




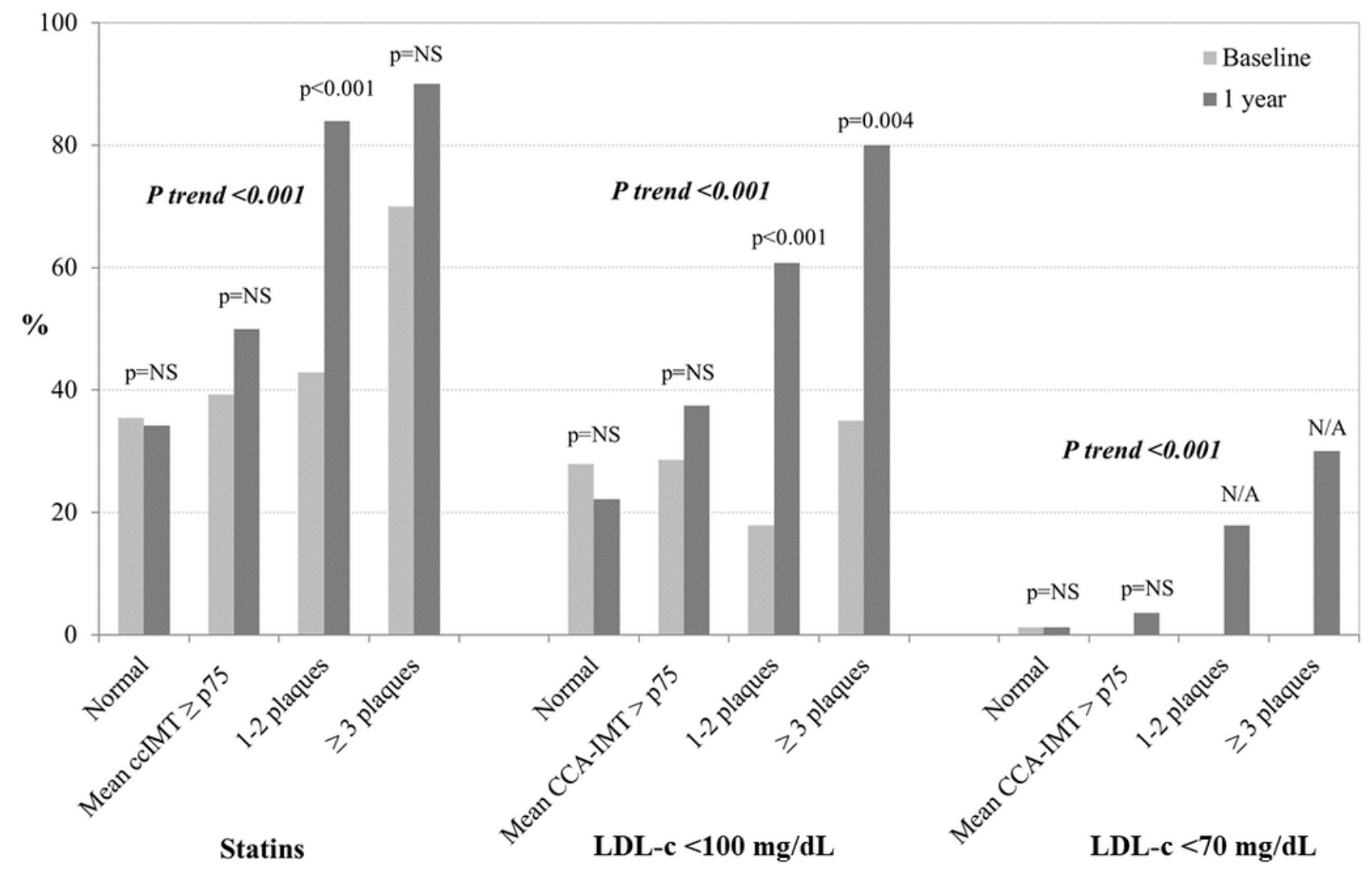

\section{Figure 1}

Patients on statins and LDL-c treatment goals according to atherosclerosis status in the US group at 1year of follow-up ( $n=211)$. P-values between baseline vs. 1-year are shown in each subclinical atherosclerosis category (for each lipid-related treatment variables). P for trend was also assessed among subclinical atherosclerosis categories at 1 year in each of the lipid-related treatment variables.

\section{Supplementary Files}

This is a list of supplementary files associated with this preprint. Click to download.

- Figuresupplem1.png

- T1DcarotidUS.SupplementarymateriaICD.docx 